University of Nebraska - Lincoln

DigitalCommons@University of Nebraska - Lincoln

2006

Pollen Concentration Analysis of Ancestral Pueblo Dietary

Variation

Karl J. Reinhard

University of Nebraska-Lincoln, kreinhard1@mac.com

Sherrian Edwards

Teyona R. Damon

University of Nebraska-Lincoln

Debra K. Meier

PathoEcology Services, dmeier1@unl.edu

Follow this and additional works at: https://digitalcommons.unl.edu/anthropologyfacpub

Part of the Anthropology Commons

Reinhard, Karl J.; Edwards, Sherrian; Damon, Teyona R.; and Meier, Debra K., "Pollen Concentration Analysis of Ancestral Pueblo Dietary Variation" (2006). Anthropology Faculty Publications. 27.

https://digitalcommons.unl.edu/anthropologyfacpub/27

This Article is brought to you for free and open access by the Anthropology, Department of at DigitalCommons@University of Nebraska - Lincoln. It has been accepted for inclusion in Anthropology Faculty Publications by an authorized administrator of DigitalCommons@University of Nebraska - Lincoln. 
Published in Palaeogeography, Palaeoclimatology, Palaeoecology 237 (2006) 92-109. Copyright 2006, Elselvier. doi:10.1016/j.palaeo.2005.11.030. http://www.elsevier.com/locate/palaeo. Used by permission.

\title{
Pollen Concentration Analysis of Ancestral Pueblo Dietary Variation
}

\author{
Karl J. Reinharda ${ }^{\mathrm{a},}$, Sherrian Edwards ${ }^{\mathrm{b}}$, Teyona R. Damon ${ }^{\mathrm{a}}$, Debra K. Meier ${ }^{\mathrm{c}}$ \\ a School of Natural Resources, University of Nebraska-Lincoln, Lincoln, NE, United States \\ ${ }^{\mathrm{b}}$ Dallas, TX, United States \\ c PathoEcology Services, Lincoln, NE, United States \\ *Corresponding author: kreinhard1@unl.edu (K.J. Reinhard).
}

Received March 24, 2003; accepted November 8, 2005.

\begin{abstract}
Previous coprolite research on the Colorado Plateau has shown that macrofossils are a useful way of statistically demonstrating prehistoric dietary variation of Ancestral Pueblos (Anasazi). Up until now, pollen concentration from human coprolites has not been used for comparative, statistical study. We present here the statistical analysis of pollen concentration values of coprolites from two Ancestral Pueblo sites, Salmon Ruin and Antelope House. The data show that although most pollen types do not show statistically significant variation, there are some types that show how different Ancestral Pueblo populations adapted to plant resources in different environments. The analysis indicates that future work should focus more on pollen concentration analysis of coprolites.
\end{abstract}

Keywords: Ancestral Pueblo; Anasazi; Palynology; Pollen concentration; Coprolites; Diet

\section{Introduction}

Minnis (1989) demonstrated that coprolite macrofossils from Ancestral Pueblo (Anasazi) sites were particularly important in identifying culturally-defined dietary patterns. "Ancestral Pueblo" refers to the prehistoric precursors of modern Pueblo societies such as the Hopi, Zuni, and Rio Grande Pueblos. Ancestral Pueblo societies were among several prehistoric groups that occupied the Colorado Plateau, a region that includes parts of Arizona, New Mexico, Colorado, and Utah. To date, no researcher has attempted to assess the value of pollen concentration analysis in defining different patterns of Ancestral Pueblo resource use at separate sites. We are taking this opportunity to evaluate the value of coprolite pollen concentration techniques in assessing variation in
Ancestral Pueblo dietary practices between two very different Ancestral Pueblo sites: Salmon Ruin, New Mexico and Antelope House, Arizona.

Antelope House in Canyon de Chelly National Monument, Arizona, and Salmon Ruin near Bloomfield, New Mexico were excavated with particular attention paid to recovery of biological remains (Figure 1). Both sites were excavated in the "New Archaeology" period in the late sixties and seventies. The focus on the scientific recovery of biological data was pioneered in the excavations of these sites. Coprolites and other biological remains from both sites have been studied (Reinhard, 1992, 1996). With regard to other remains, both are documented by monographs (IrwinWilliams and Shelley, 1980; Morris, 1986). However, Antelope House studies are more represented in journal articles and book chapters (Fry and Hall, 1975; Re- 


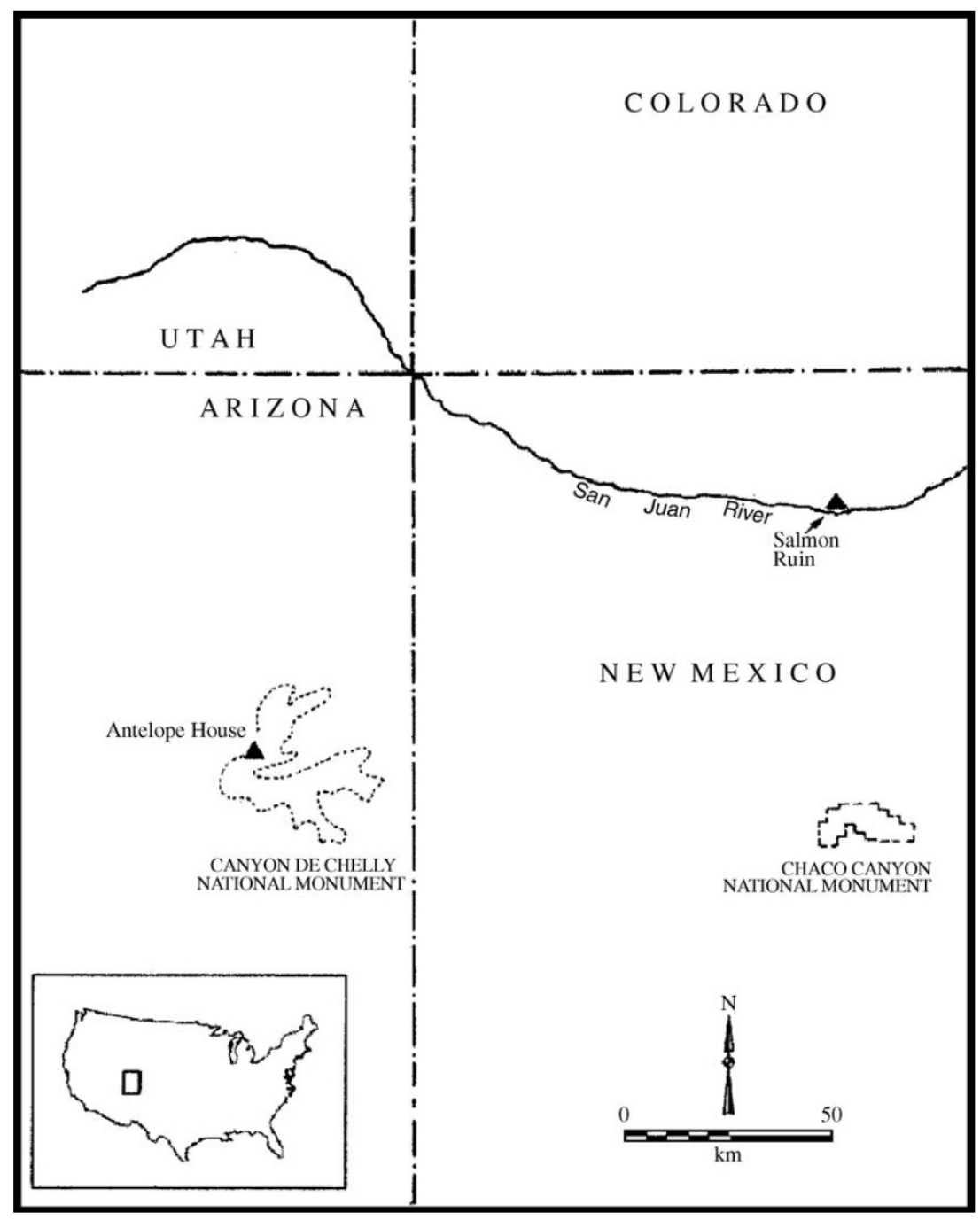

Fig. 1. Location map for Antelope House, Arizona and Salmon Ruin, New Mexico with archaeological areas mentioned in text.

inhard, 1992, 1993, for review Sutton and Reinhard, 1995; Reinhard, 1996). In 1975, volume 41 of the Kiva (Journal of the Arizona Archaeological and Historical Society) was committed to articles concerning Antelope House archaeology. Fewer articles appeared regarding the biological analysis of Salmon Ruin (Doebley, 1976; Burgess-Terrel, 1979; Doebley, 1981, 1983; Lentz, 1984). However, the methods used in the biological analysis were published in monograph form (Bohrer and Adams, 1977) as well as the results of the analysis of botanical remains from specific features (Adams, 1980). A comparative analysis of available published and unpublished data for the site was done by Reinhard (1996). Because these sites were critical in the development of Ancestral Pueblo paleoethnobotany, it is appropriate that coprolites from these sites continue to be used to develop new methods of analysis.

These are particularly good sites to compare. Both have Pueblo III Period (AD 1,100-1,300) occupations with coprolites. Approximately the same number of people lived in the Pueblo III occupations of each village (Reinhard, 1996). Both sites have coprolite deposits that can be sampled to diversify the number of individual defecations by separate Ancestral Pueblo people represented by the coprolites (Reinhard, 1996). Previous analyses of the coprolites and sites indicate that the sites were used year-round and that coprolites were deposited year-round (Williams-Dean, 1986; Sutton and Reinhard, 1995). Therefore, there is 
no evidence of differential seasonal use of the sites or the latrines at the sites.

In other ways the sites form a contrast. Antelope House is located on the floor of Canyon de Chelly. Salmon Ruin is located overlooking the flood plain of the San Juan River in open country. The Pueblo III occupation of Antelope House is the final manifestation of indigenous occupation of the site since Pueblo I times (AD 700), and since Basket Maker times for Canyon de Chelly in general, (at least since AD 300). In contrast, Salmon Ruin was originally built by the Chacoan Ancestral Pueblo as a colony. The Pueblo III occupation of Salmon Ruin is derived from a San Juan River Ancestral Puebloans that moved into Salmon Ruin after it was abandoned by the Chacoans in the beginning of the Pueblo III Period (AD 1,130). Thus, the Salmon Ruin people were new San Juan occupants and were adapted to the San Juan River environment. The Pueblo III people of Antelope House were the descendants of a tradition that had lived in Canyon de Chelly for centuries. Importantly, the cultural traditions of the sites were distinct. Antelope House was a classic Kayenta Ancestral Pueblo village adapted to the canyon country of northeastern Arizona. The Pueblo III occupation of Salmon Ruin was of the San Juan Ancestral Pueblo adapted to drier, high mesa country of the San Juan River. Therefore, the sites represent different traditions associated with different environments.

Pollen analysis has been a central part of coprolite research from the earliest studies in North America (Martin and Sharrock, 1964). For most of the history of coprolite pollen research, pollen data have been presented as percentage expression of pollen taxa present in studied coprolites. In the last decades, a newer method of presenting pollen data was applied to coprolite pollen analysis. This is the pollen concentration method that allows one to calculate the approximate number of pollen grains per unit measure of coprolites. This method was reviewed by Maher (1981). Maher presents methods of calculating the numbers of pollen grains per gram of sediment using the following formula:

Pollen concentration $=((p / m) \times e) / w$

$p$ pollen grains counted

$m$ marker grains counted

$e$ number of exotic marker pollen grains added

$w$ weight or volume of sediment

Researchers began to apply this method to human coprolites. Reinhard and colleagues (1991) used pollen concentration data to develop interpretations of me- dicinal use of certain plant taxa. They discovered that pollen concentration values are particularly compelling because they reveal that tremendous amounts of pollen were consumed by prehistoric Southwesterners. Pollen concentration values ranged into the millions of pollen grains per gram of coprolite. Such high quantities of pollen in human coprolites had been appreciated previously by only one researcher. Sobolik (1988) calculated pollen concentration values for coprolites from the lower Pecos region of Texas. She also found that human coprolites contained large quantities of pollen. She used pollen concentration values to interpret the passage of time between the consumption of pollen and the defecation of pollen. In essence, she interpreted coprolites with very high concentration values as evidence of recent consumption of pollen-rich foods and coprolites with low concentrations as evidence of pollen-rich food consumption many days before defecation. Most recently, Reinhard et al. (2002) used pollen concentration analysis in conjunction with phytolith and macroscopic analysis to reconstruct Archaic diet in the northern Sonoran Desert. They found that pollen concentration was particularly important in identifying dietary use of yucca, prickly pear, mustard family, and grass family. The pollen concentration data also demonstrate medicinal or dietary use of willow and Mormon tea.

The aim of this study is to evaluate the value of pollen concentration in defining dietary differences between two Ancestral Pueblo sites.

\section{Materials and methods}

The coprolite sampling strategies for the sites and determination of human origin were detailed by Reinhard (1996). The goal of the strategy was to diversify the samples so that many defecations by separate humans were sampled. At Antelope House, this was accomplished by taking single coprolites from several separate, discrete, and dated latrines. At Salmon Ruin, only one latrine was sampled. It was, however, a very large, stratified deposit of coprolites of which an estimated 10,000 were excavated and curated. One coprolite was taken from alternate $10 \mathrm{~cm}$ levels in alternate $1 \mathrm{~m}$ grids. The sampling was done by Reinhard and Meier.

Ultimately, 180 coprolites from Antelope House (Reinhard, 1992) and 112 coprolites from Salmon Ruin were selected for analysis. The macrofloral remains from all of these were analyzed (Sutton and Reinhard, 1995; Reinhard, 1996). All of these were analyzed microscopically for parasites before pollen processing (Reinhard, 1992). In the parasite analysis, differential diagnosis of Equisetum spores versus Populus pol- 
len was done based on the identification of elators on spores. A subsample was analyzed for phytolith content (Reinhard and Danielson, 2005). Finally, Edwards and Reinhard analyzed the pollen from 52 coprolites, 26 from each site.

Reinhard (1993) published his comparative observations of coprolite pollen recovery from very limited chemical processing to extensive chemical processing. With regard to Ancestral Pueblo coprolites from Utah, New Mexico, and Arizona, Reinhard found that equal results were achieved with both extremes.

One gram fragments of Antelope House coprolites were selected for analysis. One gram fragments from most Salmon Ruin coprolites were available for study. However, some fragments were only 0.75 or $0.5 \mathrm{~g}$. To each sample, one Lycopodium spore tablet containing 11,400 spores was added. All fragments were taken from the interior of the coprolite. The coprolites described in this paper were processed through extensive chemical treatments following Williams-Dean (1986) with one exception. The samples were rehydrated in $0.5 \%$ trisodium phosphate for $48 \mathrm{~h}$. After rehydration, macroscopic remains were screened from the microscopic remains and the microscopic residues were washed three times in distilled water. The sediments were treated in approximately $40 \%$ hydrochloric acid. After three distilled water washes, the sediments were left for $24 \mathrm{~h}$ in approximately $70 \%$ hydrofluoric acid. The samples were then washed repeatedly in distilled water until the supernatant was clear. After water washes and one glacial acetic acid wash, the residues were treated with a 20 min acetolysis treatment at $100^{\circ} \mathrm{C}$. After one glacial acetic acid wash, the samples were then washed repeatedly in distilled water until the supernatant was clear. Finally and unlike Williams-Dean (1986), the sediments were treated in $0.5 \% \mathrm{KOH}$ for 2 min and washed in distilled water three times. This was done to facilitate staining in basic fuchsin. The samples were then transferred to 1 dram vials and stored in glycerine. At least 200 pollen grains were counted for each sample, and up to 1,000 grains were counted for some samples. Pollen types were identified with reference collections of Colorado Plateau pollen samples. Single pollen grains and pollen aggregates were counted and tabulated. We noticed that many maize pollen grains were broken, shredded, or fragmented. These grains were consistent with those described by Bryant and Morris (1986) associated with grinding stones. We counted broken maize grains separately in order to determine whether there was significance in this observation. Only maize annuli were counted for the fragmented maize grains.
For statistical analysis, SAS was used for calculation of descriptive statistics, chi square values, and Wilcoxon analysis. The NPAR1WAY procedure was used to determine Wilcoxon scores (rank sums) for pollen counts by variable site.

\section{Results and analysis}

Sixteen pollen categories were chosen for statistical analysis. Whole maize, broken maize, and total maize categories were chosen to determine if there was a difference between the sites in the consumption of ground grain, assuming that broken maize grains resulted from grinding (Bryant and Morris, 1986). During macroscopic analysis, the terminal nodes immediately proximal to the strobili of Equisetum (horsetail) were found in several Antelope House coprolites (Sutton and Reinhard, 1995). Therefore, we thought it would be of interest to compare the frequency of $E q$ uisetum spores between the sites. Similarly, one Antelope House coprolite was composed of fiber with thousands of Typha latifolia pollen grains (Sutton and Reinhard, 1995). This coprolite was so rich in pollen, that it actually appeared yellow. Therefore, Typha was a logical choice for comparative pollen analysis. The macroscopic analysis showed a difference in Rhus (sumac) and Phaseolus vulgaris (bean) consumption with these plants more commonly occurring in Salmon Ruin coprolites. Phytolith analysis showed that Opuntia was more frequently eaten at Antelope House (Reinhard and Danielson, 2005). Therefore, we chose Rhus, Phaseolus, and Opuntia as comparative categories. Cleome pollen was common in the coprolites and the high frequencies of this type begged exploration. In previous analyses of coprolites, we found high spine Asteraceae to be common and therefore, we chose this category for analysis. Finally we chose to evaluate a number of anemophilous types to gain an idea of how much pollen could be ingested from the ambient environment and if it was possible to sort out dietary use of these types from ambient contamination. The anemophilous types chosen for study were low spine Asteraceae, Cheno-Am (Chenopodiaceae/ Amaranthaceae), Juniperus (juniper), Pinus (pine), and Poaceae (grass family).

The pollen concentration values for Salmon Ruin and Antelope House are presented in Tables 1 and 2 respectively. The comparative descriptive statistics for sixteen select taxa are presented in Table 3 . The first stage of the analysis was comparison of the frequency of occurrence of the categories between the sites. Chi square analysis (Table 4) showed that the frequency differences were significant at the 0.05 level only for Typha and Equisetum. Both of these taxa were more 
Table 1

Pollen concentration values for 26 coprolites from Salmon Ruin

\begin{tabular}{|c|c|c|c|c|c|}
\hline Taxa & 1 & 2 & 3 & 6 & 7 \\
\hline Apiaceae & & & & 285 & \\
\hline Artemisia & 6270 & 1524 & 276 & & \\
\hline Asteraceae-high spine & 1425 & & 69 & & 7600 \\
\hline Asteraceae-low spine & 7695 & 1368 & 622 & 1700 & \\
\hline Brassicaceae & 285 & & & & \\
\hline Carex & 285 & & & & \\
\hline Cheno-Am & 7695 & 80,256 & 8291 & 3705 & 273,600 \\
\hline Cleome & 855 & 9576 & 967 & 62,415 & 53,200 \\
\hline \multicolumn{6}{|l|}{ Cucurbita } \\
\hline Ephedra & & 456 & & & \\
\hline \multicolumn{6}{|l|}{ Equisetum } \\
\hline \multicolumn{6}{|l|}{ Eriogonum } \\
\hline Fabaceae & & 456 & & & \\
\hline Juniperus & 4560 & 456 & 69 & 570 & \\
\hline Ligulaflorae type & 285 & & & & \\
\hline \multicolumn{6}{|l|}{ Liliaceae } \\
\hline Maize broken & 855 & 1814 & 2902 & 855 & $5,145,200$ \\
\hline Maize whole & 1425 & 3192 & 898 & 855 & $1,725,200$ \\
\hline \multicolumn{6}{|l|}{ Opuntia } \\
\hline Pinus & 3420 & 1368 & 484 & & \\
\hline \multicolumn{6}{|l|}{ Plantago } \\
\hline Poaceae & 1710 & 912 & 207 & 1140 & 592,800 \\
\hline \multicolumn{6}{|l|}{ Polemonium } \\
\hline \multicolumn{6}{|l|}{ Quercus } \\
\hline \multicolumn{6}{|l|}{ Rhus } \\
\hline \multicolumn{6}{|l|}{ Rosaceae } \\
\hline Sarcobatus & 855 & & & & \\
\hline \multicolumn{6}{|l|}{ Salix } \\
\hline Shepherdia & & 456 & & & \\
\hline \multicolumn{6}{|l|}{ Sphaeralcea } \\
\hline \multicolumn{6}{|l|}{ Typha } \\
\hline Unknown & 7125 & 456 & & & \\
\hline Unidentifiable & 23,085 & 912 & 1244 & & 1520 \\
\hline Taxa & 8 & 9 & 10 & 11 & 13 \\
\hline Apiaceae & & 3800 & & & \\
\hline \multicolumn{6}{|l|}{ Artemisia } \\
\hline Asteraceae-high spine & & & 1221 & & 39,086 \\
\hline Asteraceae-low spine & 1256 & & & & \\
\hline Brassicaceae & & 30,400 & & & \\
\hline \multicolumn{6}{|l|}{ Carex } \\
\hline Cheno-Am & 2511 & & 1221 & & 19,542 \\
\hline Cleome & $1,216,000$ & $3,750,600$ & & & $6,364,457$ \\
\hline Cucurbita & & & 407 & & \\
\hline Ephedra & & & 407 & & \\
\hline \multicolumn{6}{|l|}{ Equisetum } \\
\hline \multicolumn{6}{|l|}{ Eriogonum } \\
\hline \multicolumn{6}{|l|}{ Fabaceae } \\
\hline Juniperus & & & & & \\
\hline Ligulaflorae type & & & & & \\
\hline Liliaceae & & 7600 & & & \\
\hline Maize broken & 11,300 & & 814 & 589,650 & 39,086 \\
\hline Maize whole & 25,111 & & 407 & 711,511 & 45,600 \\
\hline Opuntia & 7533 & & 401,850 & & \\
\hline Pinus & & & 407 & & \\
\hline Plantago & 2511 & 3800 & & & \\
\hline Poaceae & & & & & \\
\hline
\end{tabular}


Pollen Concentration Analysis of Ancestral Pueblo Dietary Variation

Table 1 (continued)

\begin{tabular}{|c|c|c|c|c|c|}
\hline Taxa & 8 & 9 & 10 & 11 & 13 \\
\hline \multicolumn{6}{|l|}{ Polemonium } \\
\hline Quercus & 1256 & & & & \\
\hline \multicolumn{6}{|l|}{ Rhus } \\
\hline Rosaceae & & & & 1310 & \\
\hline \multicolumn{6}{|l|}{ Sarcobatus } \\
\hline \multicolumn{6}{|l|}{ Salix } \\
\hline \multicolumn{6}{|l|}{ Shepherdia } \\
\hline \multicolumn{6}{|l|}{ Sphaeralcea } \\
\hline \multicolumn{6}{|l|}{ Typha } \\
\hline Unknown & & & 407 & & \\
\hline Unidentifiable & & 3800 & & & 6514 \\
\hline Taxa & 15 & 16 & 17 & 18 & 19 \\
\hline \multicolumn{6}{|l|}{ Apiaceae } \\
\hline Artemisia & & & 1382 & 224 & 570 \\
\hline Asteraceae-high spine & 200,018 & 814 & & 2235 & 570 \\
\hline Asteraceae-low spine & & 2443 & 1382 & 5141 & 1140 \\
\hline Brassicaceae & & & & & 950 \\
\hline \multicolumn{6}{|l|}{ Carex } \\
\hline Cheno-Am & 5182 & 4071 & 6909 & 7153 & 2850 \\
\hline Cleome & 29,018 & 56,593 & $1,355,561$ & 5365 & 78,470 \\
\hline \multicolumn{6}{|l|}{ Cucurbita } \\
\hline \multicolumn{6}{|l|}{ Ephedra } \\
\hline Equisetum & & & & & 190 \\
\hline Eriogonum & & & & 447 & \\
\hline Fabaceae & & & & & 2470 \\
\hline Juniperus & 1036 & & & 671 & 190 \\
\hline \multicolumn{6}{|l|}{ Ligulaflorae type } \\
\hline Liliaceae & & & & & 190 \\
\hline Maize broken & 133,691 & 6921 & & 3129 & 8852 \\
\hline Maize whole & 664,309 & 1629 & & 10,059 & 48,590 \\
\hline Opuntia & & & 8291 & & 40,280 \\
\hline Pinus & & 2036 & & 2459 & 190 \\
\hline \multicolumn{6}{|l|}{ Plantago } \\
\hline Poaceae & 2073 & 1221 & 9673 & 5812 & 1520 \\
\hline \multicolumn{6}{|l|}{ Polemonium } \\
\hline Quercus & & & & 447 & \\
\hline Rhus & & 2443 & & 224 & \\
\hline \multicolumn{6}{|l|}{ Rosaceae } \\
\hline Sarcobatus & 1036 & & & 447 & 570 \\
\hline \multicolumn{6}{|l|}{ Salix } \\
\hline \multicolumn{6}{|l|}{ Shepherdia } \\
\hline \multicolumn{6}{|l|}{ Sphaeralcea } \\
\hline Typha & & 407 & & & \\
\hline Unknown & & & & 2235 & 950 \\
\hline Unidentifiable & & 3257 & 1382 & 2012 & 3420 \\
\hline Taxa & 20 & 32 & 33 & 34 & 35 \\
\hline Apiaceae & & & & & 156 \\
\hline Artemisia & 283 & & & & 156 \\
\hline Asteraceae-high spine & 50,122 & 80 & 1322 & 3257 & 312 \\
\hline Asteraceae-low spine & 2073 & 400 & 330 & & 937 \\
\hline Brassicaceae & 188 & 240 & & & \\
\hline Carex & & & & & \\
\hline Cheno-Am & 5276 & 2720 & 1487 & 8686 & 1249 \\
\hline Cleome & 4522 & & 133,165 & $1,060,743$ & 11,556 \\
\hline Cucurbita & 660 & 320 & & 1086 & 2811 \\
\hline
\end{tabular}


Table 1 (continued)

\begin{tabular}{|c|c|c|c|c|c|c|c|}
\hline Taxa & 20 & 32 & & 33 & & 34 & 35 \\
\hline \multicolumn{8}{|l|}{ Ephedra } \\
\hline Equisetum & & & & & & & 312 \\
\hline \multicolumn{8}{|l|}{ Eriogonum } \\
\hline Fabaceae & & & & & & 1086 & \\
\hline Juniperus & 94 & & & & & & \\
\hline \multicolumn{8}{|l|}{ Ligulaflorae type } \\
\hline Liliaceae & & 240 & & & & & 312 \\
\hline Maize broken & 471 & 6640 & & & & 1086 & 5153 \\
\hline Maize whole & 565 & 5280 & & & & 1086 & 937 \\
\hline Opuntia & & & & & & & 156 \\
\hline Pinus & 848 & 80 & & 330 & & 1086 & 156 \\
\hline \multicolumn{8}{|l|}{ Plantago } \\
\hline Poaceae & 848 & 80 & & 165 & & & 156 \\
\hline \multicolumn{8}{|l|}{ Polemonium } \\
\hline \multicolumn{8}{|l|}{ Quercus } \\
\hline Rhus & 188 & & & & & & \\
\hline \multicolumn{8}{|l|}{ Rosaceae } \\
\hline Sarcobatus & 94 & 634 & & 496 & & 5429 & 468 \\
\hline Salix & 94 & & & & & & \\
\hline Shepherdia & 94 & & & & & & \\
\hline \multicolumn{8}{|l|}{ Sphaeralcea } \\
\hline Typha & & & & & & 2171 & \\
\hline Unknown & & & & 330 & & & \\
\hline Unidentifiable & 565 & 480 & & 496 & & 1086 & 2342 \\
\hline Taxa & 37 & 38 & 39 & & 40 & 41 & 42 \\
\hline Apiaceae & & & & & 442 & & \\
\hline Artemisia & & & 2974 & & 442 & & \\
\hline Asteraceae-high spine & & 633 & 3635 & & & & \\
\hline Asteraceae-low spine & & 1267 & 11,896 & & 2209 & & 950 \\
\hline Brassicaceae & & & & & 442 & & 1900 \\
\hline \multicolumn{8}{|l|}{ Carex } \\
\hline Cheno-Am & & 4433 & 8922 & & 5744 & 80,108 & 9500 \\
\hline Cleome & $2,926,000$ & 20,266 & 42,296 & & 412,251 & $1,472,757$ & 931,000 \\
\hline \multicolumn{8}{|l|}{ Cucurbita } \\
\hline Ephedra & & 633 & 1322 & & & & \\
\hline \multicolumn{8}{|l|}{ Equisetum } \\
\hline \multicolumn{8}{|l|}{ Eriogonum } \\
\hline Fabaceae & & 633 & & & & & \\
\hline Juniperus & & 1267 & 8261 & & & & \\
\hline \multicolumn{8}{|l|}{ Ligulaflorae type } \\
\hline Liliaceae & & & 330 & & & & \\
\hline Maize broken & 9500 & 51,933 & 661 & & 5744 & & 950 \\
\hline Maize whole & & 53,200 & 6278 & & 6186 & & \\
\hline \multicolumn{8}{|l|}{ Opuntia } \\
\hline Pinus & & & 18,835 & & 1326 & & 1900 \\
\hline \multicolumn{8}{|l|}{ Plantago } \\
\hline Poaceae & 76,000 & 633 & 12,557 & & 3977 & & 1900 \\
\hline Polemonium & & & 330 & & & & \\
\hline Quercus & & & 661 & & & & \\
\hline Rhus & & & & & 442 & & \\
\hline \multicolumn{8}{|l|}{ Rosaceae } \\
\hline Sarcobatus & & & 2643 & & 3535 & 6162 & 950 \\
\hline Salix & & & 330 & & & & \\
\hline Shepherdia & & & & & & & \\
\hline Sphaeralcea & & & & & & & \\
\hline Typha & & & & & & & \\
\hline Unknown & & & 5948 & & & & \\
\hline Unidentifiable & 4433 & & 8591 & & 5744 & 3081 & 1900 \\
\hline
\end{tabular}

The values are number of pollen grains per gram of coprolite ( $\mathrm{pg} / \mathrm{gc}$ ). 
Pollen Concentration Analysis of Ancestral Pueblo Dietary Variation

Table 2

Pollen concentration values for 26 coprolites from Antelope House

\begin{tabular}{|c|c|c|c|c|c|}
\hline Taxa & $1-11$ & $1-17$ & $1-18$ & $2-5$ & $2-14$ \\
\hline Apiaceae & & & & & 4366 \\
\hline Artemisia & 78 & 24 & & 1090 & 1213 \\
\hline Asteraceae-high spine & 26 & 264 & 1572 & & 243 \\
\hline Asteraceae-Low spine & 26 & 120 & 376,200 & 99 & 485 \\
\hline Brassicaceae & & & 786 & & 728 \\
\hline \multicolumn{6}{|l|}{ Celtis } \\
\hline Cheno Am & 312 & 528 & 1966 & 10,706 & 7519 \\
\hline Cleome & 364 & 240 & & 2974 & 18,434 \\
\hline Cucurbita & 52 & & & & \\
\hline \multicolumn{6}{|l|}{ Ephedra } \\
\hline Equisetum & 104 & & 393 & & 2426 \\
\hline Fabaceae & 26 & 24 & & & 485 \\
\hline Fraxinus & 26 & & & 198 & \\
\hline \multicolumn{6}{|l|}{ Juglans } \\
\hline Juniperus & & & 393 & & 1213 \\
\hline \multicolumn{6}{|l|}{ Liliaceae } \\
\hline Maize broken & & 24 & & 99 & \\
\hline Maize whole & & 72 & 393 & & 243 \\
\hline \multicolumn{6}{|l|}{ Opuntia } \\
\hline Pinus & 208 & 336 & & 1685 & 2668 \\
\hline Poaceae & & 48 & & 397 & \\
\hline \multicolumn{6}{|l|}{ Polemonium } \\
\hline Quercus & & 24 & & & \\
\hline Rhus & & & & 694 & 243 \\
\hline Ribes & & 576 & & & \\
\hline \multicolumn{6}{|l|}{ Rosaceae } \\
\hline \multicolumn{6}{|l|}{ Sarcobatus } \\
\hline \multicolumn{6}{|l|}{ Salix } \\
\hline \multicolumn{6}{|l|}{ Shepherdia } \\
\hline \multicolumn{6}{|l|}{ Sphaeralcea } \\
\hline Typha & 3921 & 3552 & 11,400 & 397 & 8489 \\
\hline Unknown & 338 & 216 & & 1487 & 1213 \\
\hline \multicolumn{6}{|l|}{ Unidentifiable } \\
\hline Taxa & $2-18$ & $3-8$ & $3-12$ & $3-16$ & $3-18$ \\
\hline Apiaceae & & 301 & & & \\
\hline Artemisia & 45 & 226 & & 666 & \\
\hline Asteraceae-high spine & & 150 & 2850 & 83 & 402 \\
\hline Asteraceae-low spine & 91 & 188 & & 166 & 492 \\
\hline Brassicaceae & & 38 & & & \\
\hline Celtis & 273 & & & & 179 \\
\hline Cheno-Am & 409 & 677 & 138,225 & 14,063 & 1520 \\
\hline Cleome & 136 & 4440 & 8550 & 1331 & 2146 \\
\hline Cucurbita & & & & & 1252 \\
\hline Ephedra & 91 & & & & \\
\hline Equisetum & 45 & 75 & & & 179 \\
\hline Fabaceae & 45 & 113 & & & \\
\hline \multicolumn{6}{|l|}{ Fraxinus } \\
\hline Juniperus & & 38 & & & \\
\hline \multicolumn{6}{|l|}{ Liliaceae } \\
\hline Maize broken & 363 & 150 & & & 134 \\
\hline Maize whole & 3815 & 226 & 2850 & 83 & 134 \\
\hline \multicolumn{6}{|l|}{ Opuntia } \\
\hline Pinus & 2135 & 451 & & 250 & 894 \\
\hline Poaceae & 182 & 376 & 128,250 & & 134 \\
\hline Polemonium & & & & & \\
\hline
\end{tabular}


Table 2 (continued)

\begin{tabular}{|c|c|c|c|c|c|}
\hline Taxa & $5-19$ & $6-6$ & $6-8$ & $6-13$ & $7-5$ \\
\hline Quercus & & & 1425 & & \\
\hline Rhus & 227 & & & & \\
\hline Ribes & 182 & & & & 179 \\
\hline \multicolumn{6}{|l|}{ Rosaceae } \\
\hline Sarcobatus & 45 & 75 & & & \\
\hline Salix & 136 & & & & \\
\hline Shepherdia & & 38 & & & \\
\hline \multicolumn{6}{|l|}{ Sphaeralcea } \\
\hline \multicolumn{6}{|l|}{ Typha } \\
\hline Unknown & 500 & 301 & & 250 & 447 \\
\hline Unidentifiable & 681 & & & & 224 \\
\hline Taxa & $5-2$ & $5-8$ & $5-9$ & $5-11$ & $5-16$ \\
\hline \multicolumn{6}{|l|}{ Apiaceae } \\
\hline Artemisia & & & & 760 & \\
\hline Asteraceae-high spine & 518 & 438 & & 380 & \\
\hline Asteraceae-low spine & 4145 & 2631 & & 17,480 & \\
\hline \multicolumn{6}{|l|}{ Brassicaceae } \\
\hline \multicolumn{6}{|l|}{ Celtis } \\
\hline Cheno-Am & 367,909 & 1754 & & & \\
\hline Cleome & 71,509 & 415,662 & $12,129,600$ & 366,700 & \\
\hline Cucurbita & 518 & & & & \\
\hline \multicolumn{6}{|l|}{ Ephedra } \\
\hline Equisetum & 518 & & & 18,240 & 3800 \\
\hline Fabaceae & 6218 & & & & \\
\hline \multicolumn{6}{|l|}{ Fraxinus } \\
\hline \multicolumn{6}{|l|}{ Juniperus } \\
\hline Liliaceae & 518 & & & & \\
\hline Maize broken & 1036 & & & 380 & 858,800 \\
\hline Maize whole & 1036 & & & 380 & $2,945,000$ \\
\hline \multicolumn{6}{|l|}{ Opuntia } \\
\hline Pinus & 518 & & & 760 & \\
\hline Poaceae & 22,800 & & & 1140 & \\
\hline \multicolumn{6}{|l|}{ Polemonium } \\
\hline \multicolumn{6}{|l|}{ Quercus } \\
\hline Rhus & & & 11,400 & & \\
\hline \multicolumn{6}{|l|}{ Ribes } \\
\hline Rosaceae & 1036 & & & & \\
\hline Sarcobatus & & 438 & & & \\
\hline \multicolumn{6}{|l|}{ Salix } \\
\hline \multicolumn{6}{|l|}{ Shepherdia } \\
\hline \multicolumn{6}{|l|}{ Sphaeralcea } \\
\hline Typha & & 7015 & 11,400 & & \\
\hline Unknown & 15,545 & & & & \\
\hline \multicolumn{6}{|l|}{ Unidentifiable } \\
\hline Taxa & $5-19$ & $6-6$ & $6-8$ & $6-13$ & $7-5$ \\
\hline \multicolumn{6}{|l|}{ Apiaceae } \\
\hline Artemisia & & 1200 & & 356 & \\
\hline Asteraceae-high spine & 3353 & & 600 & & \\
\hline Asteraceae-low spine & 671 & & & 178 & \\
\hline Brassicaceae & 2682 & & & & \\
\hline Celtis & & & & & \\
\hline Cheno-Am & 41,912 & 1500 & 3000 & 3028 & 34,200 \\
\hline Cleome & & 24,450 & 2600 & 23,513 & \\
\hline Cucurbita & & & & & 11,400 \\
\hline Ephedra & & & 200 & & \\
\hline Equisetum & & 300 & 159,000 & 713 & \\
\hline
\end{tabular}


Pollen Concentration Analysis of Ancestral Pueblo Dietary Variation

Table 2 (continued)

\begin{tabular}{|c|c|c|c|c|c|c|c|}
\hline Taxa & $5-19$ & $6-6$ & & $6-8$ & & $6-13$ & $7-5$ \\
\hline Fabaceae & & 600 & & & & & \\
\hline \multicolumn{8}{|l|}{ Fraxinus } \\
\hline \multirow{2}{*}{\multicolumn{8}{|c|}{$\begin{array}{l}\text { Juniperus } \\
\text { Liliaceae }\end{array}$}} \\
\hline & & & & & & & \\
\hline Maize broken & 8718 & & & 2400 & & 48,984 & \\
\hline Maize whole & 8382 & & & 11,400 & & 52,013 & \\
\hline Opuntia & & & & 600 & & & 114,000 \\
\hline Pinus & 671 & 150 & & & & 1069 & 22,800 \\
\hline Poaceae & 335 & 300 & & 1600 & & & \\
\hline \multicolumn{8}{|l|}{ Polemonium } \\
\hline \multicolumn{8}{|l|}{ Quercus } \\
\hline Rhus & & 150 & & & & & \\
\hline \multicolumn{8}{|l|}{ Ribes } \\
\hline \multicolumn{8}{|l|}{ Rosaceae } \\
\hline Sarcobatus & & & & 200 & & & \\
\hline Salix & 335 & & & & & & \\
\hline Shepherdia & & & & 200 & & & \\
\hline \multicolumn{8}{|l|}{ Sphaeralcea } \\
\hline Typha & 1006 & 300 & & 16,000 & & 178 & $100,810,200$ \\
\hline Unknown & & 1350 & & 200 & & 178 & \\
\hline \multicolumn{8}{|l|}{ Unidentifiable } \\
\hline Taxa & $7-13$ & $7-14$ & $8-2$ & & $8-13$ & $9-13$ & $9-10$ \\
\hline \multicolumn{8}{|l|}{ Apiaceae } \\
\hline Artemisia & & 903 & & & & & \\
\hline Asteraceae-high spine & & 903 & & & 1565 & & 713 \\
\hline Asteraceae-low spine & 760 & 1693 & 386 & & 447 & 570 & 356 \\
\hline Brassicaceae & & & & & 224 & & 356 \\
\hline \multicolumn{8}{|l|}{ Celtis } \\
\hline Cheno-Am & 2280 & 2257 & 386 & & 1565 & 570 & 11,044 \\
\hline Cleome & 139,080 & 3048 & 72,844 & & 15,871 & 37,620 & 326,325 \\
\hline Cucurbita & & 113 & 386 & & & & 356 \\
\hline \multicolumn{8}{|l|}{ Ephedra } \\
\hline Equisetum & & & & & 4247 & & 1069 \\
\hline \multicolumn{8}{|l|}{ Fabaceae } \\
\hline \multicolumn{8}{|l|}{ Fraxinus } \\
\hline Juniperus & & & & & 224 & & 713 \\
\hline Liliaceae & & & & & 671 & & \\
\hline Maize broken & & 1129 & & & 6259 & 1710 & 3563 \\
\hline Maize whole & & 9707 & & & 5588 & 14,250 & 10,331 \\
\hline Opuntia & 3800 & 113 & & & 224 & & \\
\hline Pinus & 760 & 564 & 580 & & 3129 & 570 & 713 \\
\hline Poaceae & 760 & 451 & & & 1565 & & 713 \\
\hline \multicolumn{8}{|l|}{ Polemonium } \\
\hline \multicolumn{8}{|l|}{ Ouercus } \\
\hline Rhus & & 226 & & & & 570 & \\
\hline \multicolumn{8}{|l|}{ Ribes } \\
\hline \multicolumn{8}{|l|}{ Rosaceae } \\
\hline \multicolumn{8}{|l|}{ Sarcobatus } \\
\hline Salix & & 113 & & & & & \\
\hline Shepherdia & & & & & 224 & & \\
\hline Sphaeralcea & & & & & & & \\
\hline Typha & & & 193 & & & 129,960 & \\
\hline Unknown & 3040 & 1242 & 773 & & 3129 & 2280 & \\
\hline Unidentifiable & & & & & & & \\
\hline
\end{tabular}

The values are number of pollen grains per gram of coprolite (pg/gc). 
Table 3

Descriptive statistics for taxa by site

\begin{tabular}{|c|c|c|c|c|}
\hline Taxa and site & Mean & $\begin{array}{l}\text { Standard } \\
\text { deviation }\end{array}$ & $\begin{array}{l}\# / \\
26\end{array}$ & $\begin{array}{l}\text { Maximum } \\
\text { concentration }\end{array}$ \\
\hline $\begin{array}{l}\text { Artemisia Salmon } \\
\text { Ruin }\end{array}$ & 552.3 & 1349.0 & 10 & 6270 \\
\hline $\begin{array}{l}\text { Artemisia } \\
\quad \text { Antelope House }\end{array}$ & 252.4 & 422.7 & 11 & 1213 \\
\hline $\begin{array}{l}\text { Broken maize } \\
\text { Salmon Ruin }\end{array}$ & $231,815.7$ & $1,008,883.4$ & 22 & $5,145,200$ \\
\hline $\begin{array}{l}\text { Broken maize } \\
\text { Antelope House }\end{array}$ & $35,913.4$ & $168,112.1$ & 15 & 858,800 \\
\hline $\begin{array}{l}\text { Whole maize } \\
\text { Salmon Ruin }\end{array}$ & $127,396.9$ & $374,700.8$ & 20 & $1,725,200$ \\
\hline $\begin{array}{l}\text { Whole maize } \\
\text { Antelope House }\end{array}$ & $117,919.4$ & $576,710.0$ & 19 & $2,945,000$ \\
\hline $\begin{array}{l}\text { Total maize } \\
\text { Salmon Ruin }\end{array}$ & $336,925.3$ & $1,348,019.0$ & 22 & $6,870,400$ \\
\hline $\begin{array}{l}\text { Total maize } \\
\text { Antelope House }\end{array}$ & $153,832.8$ & $744,715.7$ & 19 & $3,803,800$ \\
\hline $\begin{array}{l}\text { Cheno-Am } \\
\text { Salmon Ruin }\end{array}$ & $21,196.6$ & $55,516.6$ & 23 & 273,600 \\
\hline $\begin{array}{l}\text { Cheno-Am } \\
\text { Antelope House }\end{array}$ & $24,897.3$ & $75,314.7$ & 23 & 367,909 \\
\hline $\begin{array}{l}\text { Cleome Salmon } \\
\text { Ruin }\end{array}$ & $769,139.7$ & $1,489,134.7$ & 23 & $6,364,457$ \\
\hline $\begin{array}{l}\text { Cleome Antelope } \\
\text { House }\end{array}$ & $525,670.7$ & $2,369,720.7$ & 22 & $12,129,600$ \\
\hline $\begin{array}{l}\text { Cucurbita Salmon } \\
\text { Ruin }\end{array}$ & 203.2 & 590.1 & 5 & 2814 \\
\hline $\begin{array}{l}\text { Cucurbita } \\
\text { Antelope } \\
\text { House }\end{array}$ & 541.4 & 2231.3 & 7 & 11,400 \\
\hline $\begin{array}{l}\text { Equisetum Salmon } \\
\text { Ruin }\end{array}$ & 0 & 0 & 0 & 0 \\
\hline $\begin{array}{l}\text { Equisetum } \\
\quad \text { Antelope House }\end{array}$ & 7350.4 & $31,144.5$ & 14 & 159,000 \\
\hline $\begin{array}{l}\text { High spine } \\
\text { Asteraceae } \\
\text { Salmon Ruin }\end{array}$ & $12,015.4$ & $40,193.0$ & 16 & 20,0018 \\
\hline $\begin{array}{l}\text { High spine } \\
\text { Asteraceae } \\
\text { Antelope House }\end{array}$ & 540.8 & 878.4 & 16 & 3353 \\
\hline $\begin{array}{l}\text { Juniperus Salmon } \\
\text { Ruin }\end{array}$ & 660.5 & 1804.5 & 10 & 8261 \\
\hline $\begin{array}{l}\text { Juniperus } \\
\quad \text { Antelope House }\end{array}$ & 121.5 & 282.4 & 7 & 1213 \\
\hline $\begin{array}{l}\text { Low spine } \\
\text { Asteraceae } \\
\text { Salmon Ruin }\end{array}$ & 1646.5 & 2723.3 & 17 & 11,896 \\
\hline $\begin{array}{l}\text { Low spine } \\
\text { Asteraceae } \\
\text { Antelope House }\end{array}$ & $15,660.9$ & $73,616.4$ & 20 & 376,200 \\
\hline $\begin{array}{l}\text { Opuntia Salmon } \\
\text { Ruin }\end{array}$ & $17,619.6$ & $78,780.6$ & 5 & 401,850 \\
\hline $\begin{array}{l}\text { Opuntia Antelope } \\
\text { House }\end{array}$ & 4566.8 & $22,332.6$ & 5 & 114,000 \\
\hline $\begin{array}{l}\text { Pinus Salmon } \\
\quad \text { Ruin }\end{array}$ & 1343.3 & 3684.8 & 15 & 18,835 \\
\hline $\begin{array}{l}\text { Pinus Antelope } \\
\text { House }\end{array}$ & 1573.5 & 4406.9 & 20 & 22,800 \\
\hline
\end{tabular}

Table 3 (continued)

\begin{tabular}{|c|c|c|c|c|}
\hline Taxa and site & Mean & $\begin{array}{l}\text { Standard } \\
\text { deviation }\end{array}$ & $\begin{array}{l}\# / \\
26\end{array}$ & $\begin{array}{l}\text { Maximum } \\
\text { concentration }\end{array}$ \\
\hline $\begin{array}{l}\text { Poaceae Salmon } \\
\text { Ruin }\end{array}$ & $27,437.9$ & $116,264.0$ & 19 & 592,800 \\
\hline $\begin{array}{l}\text { Poaceae Antelope } \\
\text { House }\end{array}$ & 6117.4 & $25,300.7$ & 15 & 128,250 \\
\hline Rhus Salmon Ruin & 126.8 & 483.0 & 4 & 2443 \\
\hline $\begin{array}{l}\text { Rhus Antelope } \\
\text { House }\end{array}$ & 519.6 & 2226.5 & 8 & 11,400 \\
\hline $\begin{array}{l}\text { Typha Salmon } \\
\text { Ruin }\end{array}$ & 99.2 & 430.0 & 2 & 2171 \\
\hline $\begin{array}{l}\text { Typha Antelope } \\
\text { House }\end{array}$ & $3,884,769.7$ & $19,769,002.7$ & 18 & $101,000,000$ \\
\hline
\end{tabular}

common at Antelope House. Broken maize is more common at Salmon Ruin, and the difference between the sites is almost statistically significant with a $p(Z)$ value of 0.0663 . Therefore, simple examination of the frequency data reveals three interesting differences between the sites with regard to dietary use of mesic taxa and ground maize.

To determine which taxa showed significant differences between the two sites, we ran the Wilcoxon procedure through SAS for each of the pollen categories. This is a 1 way non-parametric procedure which assumes a non-normal distribution of events. The resulting Z-values of this test are presented in Table 5. Five total taxa showed significant difference at the $10 \%$

Table 4

Chi square values for taxa of interest

\begin{tabular}{llll}
\hline Taxa & Chi square & $p$ value & Significant? \\
\hline Artemisia & 0.080 & 0.7775 & No \\
Broken maize & 3.373 & 0.0663 & No \\
Whole maize & 0.103 & 0.7488 & No \\
Total maize & 0.461 & 0.4971 & No \\
Cheno-Am & 0 & 1 & No \\
Cleome & 0.165 & 0.6845 & No \\
Cucurbita & 0.108 & 0.7420 & No \\
Equisetum & 8.739 & 0.0031 & Yes \\
High spine Asteraceae & 0 & 1 & No \\
Juniperus & 0.35 & 0.5544 & No \\
Low spine Asteraceae & 0.375 & 0.5404 & No \\
Opuntia & 0 & 1 & No \\
Pinus & 1.398 & 0.2370 & No \\
Poaceae & 0.765 & 0.3819 & No \\
Rhus & 0.975 & 0.3234 & No \\
Typha & 18.281 & Less than & Yes \\
& & 0.0001 & \\
\hline
\end{tabular}


Table 5

Wilcoxon scores for taxa of interest

\begin{tabular}{lllll}
\hline Taxa & Wilcoxon score & Significant at 0.01 level & Significant at 0.05 level & Significant at 0.10 level \\
\hline Artemisia & 1.000 & No & No & No \\
Broken maize & 0.0067 & Yes & Yes & Yes \\
Whole maize & 0.1746 & No & No & No \\
Cheno-Am & 0.1696 & No & No & Yes \\
Cleome & 0.0462 & No & Yes & No \\
Cucurbita & 0.6049 & No & No & Yes \\
Equisetum & 0.0019 & Yes & Yes & No \\
High spine Asteraceae & 0.3181 & No & No & No \\
Juniperus & 0.2824 & No & No & No \\
Low spine Asteraceae & 0.4348 & No & No & No \\
Opuntia & 0.8947 & No & No & No \\
Pinus & 0.3061 & No & No & Yes \\
Poaceae & 0.0742 & No & No & No \\
Rhus & 0.3042 & Yes & Yes & \\
\end{tabular}

confidence level. Poaceae showed a relative significance of difference with Z-value of 0.0742. Cleome was significant at the $5 \%$ confidence interval with a Z-value of 0.0462. The other three taxa, Typha, Equisetum, and broken maize showed very significant differences between sites with $Z$-values less than .01. Thus, the statistical analysis shows significant variation in five of the 16 taxa of interest and shows dietary variation in the use of wild and domesticated plants.

The real power of pollen concentration data is its ability to document the magnitude of pollen ingestion. The concentration value ranges of many taxa ran into hundreds of thousands to millions of pollen grains per gram of coprolite (pg/gc). The maximum values for each taxon and site are presented in Table 3 . The highest total concentration of maize, including broken grains and whole grains, was 6,870,400 pollen grains per gram of coprolite $(\mathrm{pg} / \mathrm{gc})$ at Salmon Ruin compared to $3,803,800 \mathrm{pg} / \mathrm{gc}$ for Antelope House. In general, pollen grains from maize are more common in Salmon Ruin coprolites as seen in the higher mean $(336,925.3 \mathrm{pg} / \mathrm{gc})$ relative to the mean of $153,832.8$ $\mathrm{pg} / \mathrm{gc}$ for Antelope House. The means of whole maize pollen abundance are very similar for the sites (Table 3). However, there are four coprolites from Salmon Ruin that exceed $100,000 \mathrm{pg} / \mathrm{gc}$ as opposed to one for Antelope House. The statistically significant chi square value ( $p=0.1$ to 0.05 ) for the difference in broken maize pollen (Table 4) is amplified by the pollen concentration data. Both the mean and maximum pg/ gc values are higher for Salmon Ruin (Tables 1-3). In general, we can assume that broken pollen was consumed with pollen-bearing, maize-based foods such as stews (Sutton and Reinhard, 1995) while the highest concentrations of whole maize probably were in- gested with corn silk as suggested by Williams-Dean (1986) and Williams-Dean and Bryant (1975).

The mesic taxa, Typha and Equisetum, are very important in documenting dietary differences in the coprolite samples. The data strongly indicate that Typha was part of Antelope House diet. The mean value of $3,884,875 \mathrm{pg} / \mathrm{gc}$ and maximum value of $101,000,000$ $\mathrm{pg} / \mathrm{gc}$ clearly show that Typha pollen was eaten at Antelope House. The lower mean of 99.1 pg/gc maximum of 2,171 pg/gc for Salmon Ruin possibly reflects ambient consumption of pollen with drinking water. Typha pollen was so abundant in some coprolites, that the coprolites actually have a yellow color and examination of the macrofloral component of such coprolites revealed clumps of pollen held together by spongy fibers. Clearly, the Antelope House Ancestral Puebloans collected and ate Typha male spikes. The mean concentration values of Equisetum spores (7354.7 pg/gc for Antelope House versus 0 pg/gc for Salmon Ruin ) and maximum concentration values $(159,000 \mathrm{pg} / \mathrm{gc}$ for Antelope House versus $0 \mathrm{pg} / \mathrm{gc}$ for Salmon Ruin) support the significant chi square analysis. These data verify the macrofloral analysis which indicated that Equisetum strobili were a part of Antelope House diet.

After maize, Cleome is the most ubiquitous dietary pollen type found in Ancestral Pueblo coprolites (Martin and Sharrock, 1964; Aasen, 1984; WilliamsDean, 1986). Cleome is an insect pollinated genus that should not occur in coprolites as part of natural contamination from the ambient environment. Although the frequency of occurrence is almost the same among Salmon Ruin and Antelope House coprolites, and although the highest maximum $\mathrm{pg} / \mathrm{gc}$ occurs in an Antelope House coprolite, it appears that Cleome is 
a greater dietary pollen source at Salmon Ruin. The mean $\mathrm{pg} / \mathrm{gc}$ value is greater at Salmon Ruin and, when the data are plotted (Figure 2). It is clear that there are more Cleome values above $100,000 \mathrm{pg} / \mathrm{gc}$ for Salmon Ruin. Therefore, it is nearly ubiquitous in coprolites from both sites, but has greater concentrations at Salmon Ruin.

Cucurbita and Opuntia were prehistoric Ancestral Pueblo foods and were perhaps exploited to different degrees in different environments. Also, both types are insect pollinated and therefore should not occur as ambient contamination from the natural environment. In this analysis, neither type was ubiquitous. The difference in Cucurbita means looks important (Table 3), but it is influenced by one relatively high value of $11,400 \mathrm{pg} / \mathrm{gc}$ for one coprolite. The Opuntia values are more interesting. Each site has relatively high concentrations of this pollen type. In one Salmon Ruin coprolite, a very high value indicates the use of buds or flowers as food.

The data indicate that both high spine Asteraceae and low spine Asteraceae were background and dietary pollen sources (Table 3). With regard to pollination, low spine grains are primarily anemophilous

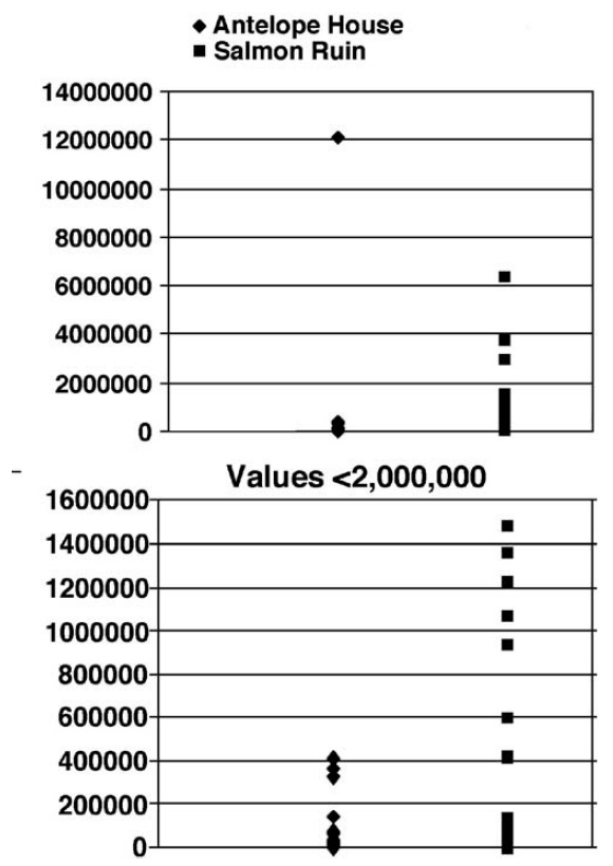

Fig. 2. Pollen concentrations for Cleome compared between Antelope House and Salmon Ruin. Although Antelope House has one very high spike, high concentration values are more ubiquitous at Salmon Ruin. while high spine grains tend to be entomophilous. Both types occur in a majority of the samples. Usually, the concentrations for these types are under 20,000 grains per gram. However, at Salmon Ruin there are high numbers of high spine Asteraceae pollen at 40,000, 50,000, and 200,000 gp/gc. These higher values suggest that Asteraceae pollen-rich foods were eaten. At Antelope House there is one high value for low spine Asteraceae at 376,200 pg/gc. This high value also suggests that Asteraceae pollen-rich foods were eaten.

Chenopodium and Amaranthus seeds were eaten at both sites as shown by macrofloral analysis (Reinhard, 1992). Chenopodium or Amaranthus greens were eaten at Antelope House but not at Salmon Ruin as shown by phytolith analysis (Reinhard and Danielson, 2005). The high concentrations of Cheno-Am pollen in a minority of coprolites from both sites show that ChenoAm pollen-rich foods were part of the diet at both sites. However, the majority of coprolites have lower concentrations of less than $10,000 \mathrm{pg} / \mathrm{gc}$. These lower values are probably the result of ingestion of ambient pollen in air, drinking water, or food contaminated with anemophilous pollen.

Rhus seeds were a common food at Salmon Ruin as shown by macrofloral analysis of coprolites (Reinhard, 1996). The pollen data show no evidence of high concentration values (Table 3). Therefore, Rhus pollen-rich foods such as flowers were apparently not eaten at the sites.

Poaceae macrofossils, excluding maize, were found in Antelope House and Salmon Ruin coprolites. These included seeds of non-cultivated grasses, and glumes from grass spikelets. Most of the pollen concentration values of wild Poaceae were low and consistent with what might be ingested with water, air, or contaminated food. However, there are high values at both sites (Table 3) that signal the consumption of Poaceae pollen-rich foods.

The anemophilous types Artemisia, Juniperus, and Pinus occurred in low concentrations of less than $25,000 \mathrm{pg} / \mathrm{gc}$ (Table 3). In the field, juniper bark was noted in association with Salmon Ruin coprolites, but was not incorporated in the coprolites. There is no evidence that Artemisia was eaten at either site. Pinyon pine nuts were eaten at both sites. Harvesting nuts from sticky pine cones may result in transfer of ambient pollen from the pine cones to hands and harvested nuts. Thus, some Pinus pollen may have been eaten inadvertently as part of collected food. However, for the most part the pollen from these types appears to be non-dietary.

In general, there was a relationship between the total pollen content of the coprolites and the number of 
plant taxa represented in the coprolites. Those coprolites with the highest over-all pollen concentration values had the fewest number of plant taxa represented by the pollen. Those coprolites that had relatively low pollen concentrations tended to have the largest number of pollen types. This is best seen in the data from Salmon Ruin. The coprolites that contained in excess of 1,000,000 grains per gram generally had an average of 5.2 taxa identified in the pollen counts. Coprolites with less than 10,000 grains per gram had an average of 11.9 taxa identified in the counts. The trend is also present in the Antelope House counts. The coprolites that contained in excess of $1,000,000$ grains per gram had an average of 3.7 taxa identified in the pollen counts. Coprolites with less than 10,000 grains per gram had an average of 13.8 taxa identified in the counts. The types that are less likely to appear in higher counts are wind pollinated, non-dietary types. Therefore, it appears that when large amounts of pollen-rich foods are eaten, the ambient pollen becomes infrequent relative to dietary types. This is identical to the pattern previously reported by Reinhard et al. (2002). Therefore, the likelihood of finding the more dilute ambient types is lessened in pollen-rich coprolites.

We believe that the range of values for the taxa is striking and indicates that some taxa are more sensitive to differences in prehistoric behavior than others. The general Ancestral Pueblo reliance on maize and sporadic use of squash and prickly pear, for example, makes these taxa poor indicators of prehistoric differences in resource use. However, Typha, Equisetum, Cleome, wild grass, and broken maize exhibit significant variation which characterizes these taxa as a more interesting taxa for comparison. With regard to environmental taxa such as Juniperus, Pinus, and Rhus, there is no detectable difference.

\section{Discussion}

We believe that the pollen concentration values do provide exciting comparative data. The data are strongly influenced by the prehistoric practice of eating pollen-rich food. Past and current research shows some of the sources of this pollen. Bohrer (1981) demonstrated that some wild seeds commonly eaten in prehistory carry pollen from the source plant. Therefore, the consumption of certain seeds is a source of dietary pollen. This study shows that other pollen-rich foods included florets, buds and/or flowers, strobili, and male floral spikes. With regard to the Ancestral Pueblo, wild grass florets were eaten. The buds and/ or flowers of Asteraceae, Cleome, Opuntia, and possibly Cucurbita were eaten. The strobili of Equisetum and the male floral spikes of Typha were eaten. It is likely that eating the greens of certain species could also be a source of dietary pollen. Phytolith analysis shows that wild grasses, Cleome, Chenopodium and/or Amaranthus were sources of greens for the Ancestral Pueblo at Antelope House. Therefore, some pollen could have been introduced from pollen contaminated greens. Maize pollen is abundant in the coprolites. The high concentration values which range into the millions indicate that the male florets and tassels were harvested and eaten. Another source of maize pollen could have been from ground grain as indicated by the higher amounts of broken grains at Salmon Ruin.

This study shows that pollen-rich foods were a common aspect of Ancestral Pueblo cuisine. Of the 51 coprolites studied here, $33(63 \%)$ had over 100,000 pg/ gc. Eighteen (35\%) had between 100,000 and 1,000,000 $\mathrm{pg} /$ gc. Thirteen $(25 \%)$ had between $1,000,000$ and $10,000,000 \mathrm{pg} / \mathrm{gc}$. Two coprolites $(4 \%)$ had over $10,000,000 \mathrm{pg} / \mathrm{gc}$. With regard to the dietary behavior represented by these coprolites, it appears that pollenrich foods were more important at Salmon Ruin than Antelope House. Eighteen (69\%) of Salmon Ruin coprolites have values over $100,000 \mathrm{pg} / \mathrm{gc}$ in contrast to $13(50 \%)$ of Antelope House coprolites. However, the Antelope House Ancestral Pueblo targeted pollen or spore producing plant organs for harvest, specifically Equisetum and Typha.

The next logical question is what was the nutritional benefit of pollen-rich foods? The nutritional value of pollen has been evaluated in several studies (Herbert and Shimanuki, 1978; Schmidt and Schmidt, 1984). Pollen is $44 \%$ carbohydrate, $24 \%$ protein, and is a source of fat, sodium, vitamin C, calcium, iron, and potassium. Therefore, pollen-rich foods augmented the Ancestral Pueblo dietary sources of these nutrients. The contribution of pollen-rich foods to Ancestral Pueblo diet supports Cummings (1994) assertion that Ancestral Pueblo diet was essentially healthy.

Future research needs to be done to quantify the amount of pollen present in purported food sources. There is a need to harvest wild inflorescences, seeds, and other potential pollen-rich foods to determine how many pollen grains are produced per flower or are present per gram of greens and seeds. Once these types of baseline data are collected, we can then determine how much pollen-rich food originally consumed is represented by pollen grain per gram of coprolite values. This will further elucidate the prehistoric Ancestral Pueblo dietary use of pollen. Also, further analysis of many more coprolites from these sites must be done to assess potential differences in consumption of less common types such as Apiaceae, Brassicaceae, and Liliaceae. 
The value of holistic coprolite analysis and pollen concentration can be demonstrated by contrasting past and current study. Antelope House human coprolites had been extensively analyzed decades ago. This provides us with an opportunity to show what can be learned through pollen concentration analysis in contrast to previous studies. Also, we can address the advantage of doing pollen concentration analysis as part of a holistic analysis of coprolites which includes macro-floral and phytolith analyses as well as observations of simple fecal smears made directly from rehydrated coprolites. Previous pollen analysis of Antelope House Ancestral Pueblo coprolites was done by Williams-Dean and Bryant (Williams-Dean, 1975; Williams-Dean and Bryant, 1975; WilliamsDean, 1986). The macrofloral analysis of the coprolites was done by Fry and Hall $(1975,1986)$.

The past pollen analysis and macrofloral analysis of Antelope House coprolites were done independently by separate researchers who were apparently not in communication. This resulted in misidentification of spores and a failure to recognize the dietary value of pollen and spores. Williams-Dean (1986) in her final report correctly recognized that Typha pollen was consumed by Antelope House Ancestral Pueblo. However, she confused Equisetum with Populus.It may seem outrageous that confusion of spores from a "primitive" vascular plant with pollen grains from a tree could occur. However, the spores of Equisetum and the pollen grains of Populus are very similar after acetolysis. After acetolysis, the elators which are diagnostic of Equisetum spores are destroyed (Kapp, 1969:65,67). Therefore, Equisetum spores look like Populus pollen grains after processing.

The first hint that Equisetum and not Populus was eaten comes from the macrofloral analysis. Fry and Hall found the terminal nodes immediately proximal to the strobili of Equisetum (horsetail) in 7\% of 91 coprolites (Fry and Hall, 1986). However, Fry and Hall (1986) identified the remains as "horsebrush stem" which did not indicate clearly that the strobili were eaten. Thus, the palynologists were not alerted to the presence of Equisetum strobili and consequently misidentified these structures as Populus. Had the palynologists been directly aware of the macrofloral remains, they would probably have considered the differential diagnosis of Equisetum versus Populus. A second hint that Populus might not be the correct identification could have come from Bryant and Wier's (1986) analysis of pollen from Antelope House floors. They did not find that ambient Populus pollen was abundant in any of their samples. When we began this analysis, we had the benefit of reading Fry and Hall (1975, 1986). Also, Reinhard $(1992,1996)$ had done an inde- pendent macrofloral and parasite analysis of 112 coprolites from Salmon Ruins (Reinhard, 1996) and 180 coprolites from Antelope House (Sutton and Reinhard, 1995). We also had the advantage of having 180 parasite preparations from Antelope House coprolites and 112 from Salmon Ruin coprolites. These were not processed with acetolysis solution.

Reinhard found macrofloral Equisetum remains in 10 of 180 coprolites from Antelope House but not in any Salmon Ruin coprolite. Harlan and Dennis (1986) report three species of Equisetum from the area near Antelope House, E. arvense, E. hyemale, and E. laevigatum. Reinhard compared the macrofloral Equisetum remains with modern Equisetum and discovered that these were not just stem fragments as described by Fry and Hall (1986). These modern Antelope House species noted by Harlan and Dennis (1986), like other Equisetum species, have jointed aerial stems. For these species, nodes proximal to the terminal node connect two stem sections. Therefore, stem fragments have nodes attached to two distinct stem sections. The Antelope House Equisetum nodes were definitely terminal nodes. There was no distal stem section at the node. Also, the stems proximal to the nodes were cleanly cut. This shows that the Antelope Ancestral Puebloans used sharp implements, probably stone knives to cut the plant stems just at the terminal nodes and strobili.

Another hint that the Antelope House Ancestral Puebloans ate Equisetum spores came from the fecal preparations for parasite analysis. For parasite preparations, no chemical processing beyond rehydration is done. Therefore, it was possible to examine Equisetum/Populus-like structures for elators that occur on Equisetum but not Populus. At Antelope House, we could identify the elators on some of spores in the parasite preparations but not in the Salmon Ruin preparations. We are certain that the Equisetum/Populuslike structures in the Antelope House coprolites are spores of Equisetum.

Finally, there is negative evidence from the phytolith analysis that indicates that Equisetum stems were not eaten. Equisetum stems contain phytoliths. Had stems been eaten as identified by Fry and Hall (1986), we would have found Equisetum phytoliths in the coprolites. Although phytoliths were abundant in Antelope House coprolites, no Equisetum phytoliths were found (Reinhard and Danielson, 2005).

The error by Williams-Dean $(1975,1986)$ and Williams-Dean and Bryant (1975) was probably also made by Bryant and Morris (1986). Bryant and Morris analyzed pollen samples from grinding stones and ceramic jars in comparison to several control samples. They identified Populus pollen in 28 samples from ce- 
ramic vessels, but did not encounter this type in any samples from grinding stones. The percentage of Populus pollen in the vessels ranged from $1 \%$ to $39.5 \%$ with a mean of $7.6 \%$. Of seven control samples, only two contained Populus pollen in percentages of $2 \%$ and $5 \%$. In the light of the discovery of Equisetum spores in the coprolites, it is probable that some or most of the "Populus" pollen found in the Antelope House ceramic vessels were actually Equisetum spores. It is interesting that the Populus pollen and Typha pollen was found in ceramic cemetery offerings (Bryant and Morris, 1986). Apparently, pollen and spore food sources were sufficiently valued to be included as burial offerings.

These comments are not intended to demean the work of Bryant, Wier, and Williams-Dean. Their combined Antelope House work is a milestone in the development of archaeological methods. We present this critique only to highlight that even the best palynologists can make errors when working independently of other investigators, especially analysts working with macrofossils. Because of the independence of macrofossil and pollen analysis done previously, and because of the identification of Equisetum terminal nodes as "horsebrush stem," the palynologists were not aware that a differential diagnosis of Equisetum spores and Populus pollen grains was necessary for true reconstruction of Antelope House diet. We recommend that palynologists work directly with macrofloral remains to avoid such errors. Also, we recommend that palynologists examine simple fecal smears from rehydrated coprolites to aid in differential diagnosis. Although Kapp (1969) asserts that Equisetum elators are lost in the process of fossilization, we were able to identify a few of these on spores before pollen processing. This indicates that fossilization of desiccated coprolites does not destroy the elators.

In the future, pollen concentration should be done with human coprolites from all cultural contexts especially hunter-gatherers. Hunter-gatherers probably ate pollen and spore producing organs. Heizer and Napton (1969) found this to be true of huntergatherers from the Great Basin. They note (1969:566), "dozens of the Lovelock coprolites are composed almost entirely of cattail pollen." Our analysis shows that such qualitative observations can be quantified with application of the pollen concentration technique. Only when this method is widely applied, will the anthropological community become aware of how widespread prehistoric people relied on pollen and spore producing plant organs for dietary use.

\section{Acknowledgements}

We thank the U.S. National Park Service for making available their extensive collections of coprolites housed at the Western Archaeological and Conservation Center in Tucson, Arizona. Curator Gloria Fenner was especially helpful in guiding us through the collections. WACC Curatorial Assistant, Mary Sherry, was also extremely helpful in the early days of this work and was missed in the latter days. We also thank the Salmon Ruins Museum and Research Library for providing access to the Salmon Ruin collections. Executive Director, Larry Baker, and Preservation Archaeologist, Paul Reed have been particularly helpful both on providing access to Salmon Ruin coprolite collections and also in providing guidance in writing the background sections to this analysis. Baker and Reed have been tireless in obtaining funding to preserve the tens of thousands of artifacts excavated from Salmon Ruin.

The two archaeologists who excavated Antelope House and Salmon Ruin are paragons of scientific archaeology. Don Morris excavated Antelope House with the goal of collecting all biological remains. Therefore, his excavations produced an unprecedented collection of Pueblo cave biological materials. He fostered new technology and new applications, as exemplified by this study. For those of us who worked with Cynthia Irwin-Williams in the Salmon Ruin excavations, there is universal appreciation for her scientific zeal. As a mentor in field archaeology and research design, she was an inspiration that drove many young careers. Like Morris, Irwin-Williams was an excellent scientist with a vision of the future of archaeology and the skills and tools that would come as archaeology developed as a scientific field. Both Morris and Irwin-Williams took a direct interest in the analysis of coprolites.

In the first stage of laboratory analysis, Dr. Richard Hevly of the Department of Biological Sciences, Northern Arizona University played an important role. He was a product of a unique period in Arizona archaeology when botanists were trained in the archeological and geological sciences and went on to train a generation of environmental archaeologists. He was a pioneering coprolite analyst. As a master's major professor, he gave generously of his time to teach Colorado Plateau archaeobotany. In Hevly's case, his department never fully appreciated his work in geology and archeology because it fell out of the bioscience mainstream. Therefore, his efforts in training geoscientists and archaeobotanists were largely unrewarded. 
In the second stage of analysis, Vaughn Bryant, Jr. of the Department of Anthropology at Texas A\&M University played the key role. Vaughn worked with Texas archaeologists in developing research designs for the recovery of biological remains from cave sites. Trained as a botanist, Vaughn became the chair of the A\&M Anthropology Department. In that position, he developed a center for training environmental archaeologists working in a diversity of places, including the Colorado Plateau. Vaughn directed the first palynological studies of Antelope House artifacts and coprolites. He provided the expertise, laboratory, and graduate student colleagues that contributed to the success of research projects. As graduate students, two other people contributed directly to this research. John Jones helped Reinhard master Colorado Plateau palynology. Richard Holloway encouraged the use of pollen concentration in the analysis of Antelope House.

We would like to thank the reviewers, Vaughn Bryant, Jr. and Alwynne B. Beaudoin. We would also like to thank Owen Davis for organizing the Faeces Facies Symposium and for his editorial guidance in preparing this manuscript.

\section{References}

Aasen, D.K., 1984. Pollen, macrofossil, and charcoal analyses of basketmaker coprolites from Turkey Pen Ruin, Cedar Mesa, Utah. M.A. Thesis, Department of Anthropology, Washington State University, Pullman, Washington, USA.

Adams, K.R., 1980. Pollen, Parched Seeds and Prehistory: a Pilot Investigation of Prehistoric Plant Remains from Salmon Ruin, a Chacoan Pueblo in Northwestern New Mexico. Contributions in Anthropology, vol. 9. Eastern New Mexico University, Portales, NM. Bohrer, V.L., 1981. Methods of recognizing cultural activity from pollen in archeological sites. Kiva 46, 135-142.

Bohrer, V.L., Adams, K.R., 1977. Ethnobotanical Techniques and Approaches at Salmon Ruin, New Mexico. Eastern New Mexico University Contributions in Anthropology, vol. 8. Eastern New Mexico University, Portales, NM. 220 pp.

Bryant Jr., V.M., Morris, D.P., 1986. Uses of ceramic vessels and grinding implements: the pollen evidence. In: Morris, D.P. (Ed.), Archeological Investigations at Antelope House. National Park Service Publications in Archaeology, vol. 19. National Printing Office, Washington, D.C., pp. 489-500.

Bryant Jr., V.M., Wier, G.H., 1986. Pollen analysis of floor sediment samples: a guide to room use. In: Morris, D.P. (Ed.), Archeological Investigations at Antelope House. National Park Service Publications in Archaeology, vol. 19. National Printing Office, Washington, D.C., pp. 58-71.

Burgess-Terrel, M.E., 1979. A study of Cucurbita material from Salmon Ruin, New Mexico. M.A. Thesis, Department of Anthropology, Eastern New Mexico University, Portales.

Cummings, L.S., 1994. Anasazi diet: variety in the Hoy House and Lion House coprolite record and nutritional analysis. In: Sobolik, K.D. (Ed.), Paleonutrition: the Diet and Health of Prehistoric Americans, Occasional Paper, vol. 22. Center for Archeological Investigations, Southern Illinois University at Carbondale, Carbondale, IL, pp. 134-150.

Doebley, J.F., 1976. A preliminary study of wild plant remains recovered by flotation at Salmon Ruin, New Mexico. M.A. Thesis, Department of Anthropology, Eastern New Mexico University, Portales.

Doebley, J.F., 1981. Plant remains recovered by flotation from trash at Salmon Ruin, New Mexico. Kiva 46, 169-187.

Doebley, J.F., 1983. Maize variability and cultural selection at Salmon Ruin, New Mexico. Kiva 49, 19-37.

Fry, G., Hall, H.J., 1975. Human coprolites from Antelope House: preliminary analysis. Kiva 41, 87-96.

Fry, G., Hall, H.J., 1986. Human coprolites. In: Morris, D.P. (Ed.), Archeological Investigations at Antelope House. National Park Service Publications in Archaeology, vol. 19. National Printing Office, Washington, D.C., pp. 165-188.

Harlan, A., Dennis, A.E., 1986. A preliminary plant geography of Canyon de Chelley National Monument. In: Morris, D.P. (Ed.), Archeological Investigations at Antelope House. National Park Service Publications in Archaeology, vol. 19. National Printing Office, Washington, D.C., pp. 72-83.

Heizer, R.F., Napton, L.K., 1969. Biological and cultural evidence from prehistoric human coprolites: the diet of prehistoric Great Basin Indians can be reconstructed from desiccated fecal material. Science 165, 563-568.

Herbert Jr., E.W., Shimanuki, H., 1978. Chemical composition and nutritive value of bee-collected and bee-stored pollen. Apidologie 9, 33-40.

Irwin-Williams, C., Shelley, P.H., 1980. Investigations at the Salmon Site: the structure of Chacoan Society in the Northern Southwest (Vols. I-IV). Eastern New Mexico University, Portales.

Kapp, R.O., 1969. How to Know Pollen and Spores. W.C. Brown Co., Dubuque. 249 pp.

Lentz, D.L., 1984. Utah juniper (Juniperus osteosperma) cones and seeds from Salmon Ruin, New Mexico. J. Ethnobiol. 4, 191-200.

Maher Jr., L.J., 1981. Statistics for microfossil concentration measurements employing samples spiked with marker grains. Rev. Palaeobot. Palynol. 32, 153-191.

Martin, P.S., Sharrock, F.W., 1964. Pollen analysis of prehistoric human feces: a new approach to ethnobotany. A new approach to ethnobotany. Am. Antiq. 30, 168-180.

Minnis, P., 1989. Prehistoric diet in the northern Southwest: macroplant remains from Four Corners feces. Am. Antiq. 54, 543-563.

Morris, D.P., 1986. Archeological Investigations at Antelope House. National Park Service Publications in Archaeology, vol. 19. National Park Service, Washington, D.C. 581 pp.

Reinhard, K.J., 1992. Patterns of diet, parasitism, and anemia in prehistoric west North America. In: Stuart-Macadam, P., Kent, S. (Eds.), Diet, Demography, and Disease: Changing Perspectives on Anemia. Aldine de Gruyter, New York, pp. 219-258.

Reinhard, K.J., 1993. The utility of pollen concentration in coprolite analysis: expanding upon Dean's comments. J. Ethnobiol. 9, 31-44.

Reinhard, K.J., 1996. Parasite ecology of two Anasazi villages. In: Reitz, E.J., Newson, L.A., Scudder, S.J. (Eds.), 
Case Studies in Environmental Archaeology. Plenum Press, New York, pp. 175-189.

Reinhard, K., Danielson, D.R., 2005. Pervasiveness of phytoliths in prehistoric southwestern diet and implications for regional and temporal trends for dental microwear. J. Archaeol. Sci. 32, 981-988.

Reinhard, K.J., Hamilton, D.L., Hevly, R.H., 1991. Use of pollen concentration in paleopharmacology: coprolite evidence of medicinal plants. J. Ethnobiol. 11, 117-134.

Reinhard, K., Daniels, M., Danielson, D.R., Chaves, S.M., 2002. Multidisciplinary coprolite analysis. In: Geib, P.R., Keller, D.R. (Eds.), Bighorn Cave: Test Excavation of a Stratified Dry Shelter, Mojave County, Arizona. Bilby Research Center Occasional Papers, vol. 1. Northern Arizona University, Flagstaff, pp. 135-152.

Schmidt, J.O., Schmidt, P.J., 1984. Pollen digestibility and its potential nutritional value. Glean. Bee Cult. 112, 320-322.
Sobolik, Kristin D., 1988. The importance of pollen concentration values from coprolites: an analysis of southwest Texas samples. Palynology 12, 201-221.

Sutton, M.Q., Reinhard, K.J., 1995. Cluster analysis of the coprolites from Antelope House: implications for Anasazi diet and cuisine. J. Archeol. Sci. 22, 741-750.

Williams-Dean, G., 1975. Pollen analysis of prehistoric human coprolites from Antelope House Ruin, Canyon de Chelly National Monument, Arizona. M.S. Thesis, Department of Biology, University of Texas, Austin, USA.

Williams-Dean, G., 1986. Pollen analysis of human coprolites. In: Morris, D.P. (Ed.), Archeological Investigations at Antelope House. National Park Service Publications in Archaeology, vol. 19. National Printing Office, Washington, D.C., pp. 189-205.

Williams-Dean, G., Bryant Jr., V.M., 1975. Pollen analysis of human coprolites from Antelope House. Kiva 41, 97-111. 